\title{
An Extended Scope of Practice for Audiology: the Case for Ireland
}

\author{
Amr El Refaie* \\ Department of Speech and Hearing Sciences, University College Cork (UCC), Ireland
}

*Corresponding author: Amr El Refaie, Department of Speech and Hearing Sciences, University College Cork (UCC), Ireland.

Received Date: January 15, 2020

Published Date: January 29, 2020

\section{Introduction}

There is a growing demand in the medical field for nonphysician health care professionals to provide an array of services that would help relieve the extreme pressure of long waiting lists in health services, as well as help improve the patient journey and outcomes of management. There have been moves to extend the scope of practice for nursing [1], Speech and language therapy [2,3] as well as other areas of clinical therapy over the years. Hearing services are no different. There are many studies that demonstrate the potential of what highly trained, specialized audiologists can do to contribute, for example in paediatric audiology [4] as well as to specialist balance and tinnitus clinics.

In Ireland, a review of hearing services was conducted a decade ago, and the results were published by the Health Service Executive (HSE) [5]. One of the main recommendations was the establishment of an Irish training pathway that would make the entry level into the profession of Audiology a master level qualification. Five years later a master course has been established at University College Cork. To date three cohorts of highly skilled audiologists have entered the profession.

Ear, Nose and Throat health services in Ireland have come under chronic and extreme pressure for some time. This has led to long waiting lists of non-emergency patients waiting to see a consultant for periods extending to 4 years in some areas of the country. The question is how many of those patients referred by their GPs actually need to see a consultant, given that there are no direct routes of referral to audiology services from GPs to many patients? And what needs to be done to improve the patient's journey and decrease the time needed for the patient to get help for their complaints?
ENT Audiology Waiting List Initiative, the South Infirmary, Cork

In 2018, the South Infirmary Victoria University Hospital (SIVUH), Cork, Ireland, established an initiative to tackle long ENT waiting lists. Audiology-led clinics were tasked with seeing all patients referred to ENT consultant first. A protocol for governance was established, which allowed a qualified audiologist to examine the patients, and decide whether a patient can be safely discharged to the source of referral with the Audiological assessment report, referred to Audiological management, or whether a patient needs to be seen by the ENT consultant. Details of the protocol of governance and results will be published (O Sullivan et al, in preparation). Results obtained at the end of 2018 showed that from a total of 1,318 direct referral patients seen by the audiology-led clinic, $12 \%$ were referred for full Audiological management, $10 \%$ needed further investigations, including MRI scan, $49 \%$ were discharged, while only $29 \%$ needed to be seen by the ENT consultant. Further analysis up to the end of 2019 showed very similar results in all categories. There is a drive to extend similar audiology-led clinics in other areas in Ireland at present.

What Needs to be Done to Facilitate an Extended Scope of Practice for Audiology in Ireland?

In my opinion, there is an overwhelming case for an extended scope of practice for Audiologists in the Republic of Ireland. The timing is right, not only due to the current need and long waiting lists, but also because I believe there is a strong foundation for such a development at the present time. One of the important foundations is the availability of a qualification route at master level that is designed with the needs of the Irish health services in mind. The 
program has a strong emphasis on areas identified as priorities by stake holders, namely Paediatric Audiology, Diagnostic audiology, Balance assessment and management and Tinnitus management, as well the traditional Audiology interest of aural rehabilitation and hearing assessment. The program aims to develop clinical audiologists, who are not only efficient in preforming and interpreting hearing tests, but on taking a full clinical history, using the results of investigations to formulate a differential diagnosis and decide on the need for further investigations before referring patients for medical assessment if needed. In the meantime, a post qualification route for training in specialized areas (Adult diagnostics, Paediatric assessment, Balance assessment and management, Tinnitus management, Cochlear implants and Communication in the elderly) has also been established to ensure continuous professional education and future-proofing knowledge and skills for Audiologists who decide to specialize in within the extended scope.

\section{The future}

I can see three immediate areas that can benefit from audiologyled specialized clinics in Ireland, using a similar governance structure to the one established by the South Infirmary Victoria University Hospital

1. Adult diagnostic assessment clinic for patients with hearing complaints.

2. Audiology-led Balance assessment clinics.

3. Audiology-led tinnitus assessment and management clinic.

Audiology-led diagnostic clinics would triage patients referred to ENT services, and can also perform full clinical assessment, including ordering investigations like MRI scans when indicated. That would mean that the patient who needs to see an ENT consultant, would do so with a full clinical report and investigations ready.
I strongly believe that such development is needed, and crucially that Audiology can contribute significantly to the efficiency of services, make them more fit for purpose and costeffective, and avoiding undue delays in patients receiving medical attention. I also believe that such a pathway would improve the outcomes of the patient journey as a whole. In order to make that a reality, there are still a lot of steps that need to be undertaken. From investment of the health services in the area of audiology, investment training, recruitment and improvement of working condition for Audiologists, as well as working on the establishment of strong governance and guidelines to ensure best practice principles are adhered to and ensure the safety of patients and clinicians involved. Delivering a state of the art, evidence-based diagnostic audiological services will need investment in building the required infrastructures for the profession to live in. The case to start, for me, has been overwhelmingly established.

\section{Acknowledgement}

I would like to thank Professor Nicole Muller, UCC, Dr Siobhan Laoide-Kemp for their help in the preparation of this article.

\section{Conflict of Interest}

No conflict of interest.

\section{References}

1. Cant R, Birks M, Porter J, Jacob E, Cooper S (2011) Developing advanced rural nursing practice: A whole new scope of responsibility. Collegian 18: 177-182.

2. NARG report.

3. Pokorny M, Wayne W, Thorne P, Whitfield B (2018) Is an advanced audiology-led service the solution to the paediatric ENT outpatient waiting list problem? Speech Language and Hearing 22(3): 137-141.

4. Seabrook M, Schwarz M, Ward E, Whitfield B (2019) Implementation of an extended scope of practice speech-language pathology allied health practitioner service: An evaluation of service impacts and outcomes. Int J Speech Lang Pathol 21: 65-74.

5. Ward E (2019) Elizabeth Usher memorial lecture: Expanding scope of practice-inspiring practice change and raising new considerations. Int J Speech Lang Pathol 21: 228-239. 